\title{
La dinámica espacio-temporal del Patrimonio Mundial en Peligro
}

\section{Concepción Foronda Robles ${ }^{a}$ y María Rojas Pavón ${ }^{a}$}

${ }^{a}$ Facultad de Turismo y Finanzas, Universidad de Sevilla, c/ San Francisco Javier s/n, 41018 Sevilla. foronda@us.es, marrojpav@alum.us.es.

\begin{abstract}
Resumen
Los lugares Patrimonio Mundial están sufriendo cambios, bien por el cambio climático y sus efectos como por el papel que desempeña el ser humano, responsable de la situación de emergencia que vive el planeta.

El objetivo del trabajo es analizar desde una perspectiva espacio-temporal (1979-2018) diversos aspectos concretos de la Lista en Peligro del Patrimonio Mundial. El análisis se hará a través de variables que van desde la composición de la lista y su distribución continental, el desarrollo de los países y la posesión de esta figura, el periodo de permanencia en la Lista y las asistencias económicas que la UNESCO ofrece a los mismos. Para este estudio, se han empleado State of Conservation Reports de la UNESCO, documentos de inscripción y diferentes informes generados de convenciones para la protección del patrimonio.
\end{abstract}

Los resultados demuestran el aumento del peligro en los últimos años para los Patrimonios Mundiales y las diferencias en la gestión de los mismos en función del desarrollo del país en el que se encuentran así como la propensión a localizarse en África y Oriente Medio y a pasar largos periodos de permanencia en esta "Lista Negra".

Palabras clave: Patrimonio Mundial en peligro, amenazas, acomodamiento, ayudas, conservación, desarrollo sostenible, cultura.

\footnotetext{
Abstract

World Heritage sites are suffering changes, due to climate change and its effects, as well as the role played by human being, responsible for the emergency situation that the planet is experiencing.

The aim of this work is to analyze from a space-time perspective (1979-2018) various specific aspects of the List of World Heritage in Danger. The analysis will be done through variables such as the composition of the list and its continental distribution, the development of the countries and the possession of this figure, the period of permanence in the List and the economic assistance that UNESCO offers to them. For this study, it has been used the UNESCO State of Conservation Reports, registration documents and different reports generated from conventions for the protection of heritage.

The results show the increase of the danger in the last years for the World Heritage and the differences in their management depending on the development of the country in which they are as well as the propensity to be located in Africa and the Middle East and to spend long periods of permanence in "this Black List".
}

Keywords: World Heritage in danger, threats, accommodation, assistances, conservation, sustainable development, culture. 


\section{Introducción}

Los lugares Patrimonio Mundial sufren diversas amenazas. Sin duda, las referidas al cambio climático y sus efectos son las que ejercen y ejercerán mayor presión sobre estos patrimonios (Harvey y Perry, 2015), comprometiendo incluso la continuidad de especies autóctonas, la contaminación de los océanos, la desertificación o la intensidad de desastres naturales que se ciernen sobre estos espacios naturales y culturales frágiles y vulnerables (UNESCO, 2017). La mayoría de los sitios del Patrimonio Mundial tienen algún nivel de amenaza por el cambio climático, bien amenazas potenciales o peligro real. Aunque es cierto que los ciclos naturales siempre han afectado a algunos Patrimonio Mundial, el cambio climático inducido por el hombre se ha agravado en las últimas décadas (Allan et al., 2017; Hall et al., 2016; Maus, 2014).

La dinámica espacio temporal del Patrimonio Mundial ha pendulado entre la preservación y el cambio. Los sitios naturales y culturales se enfrentan a grandes desafíos por los impactos humanos. Las discusiones van dirigidas a identificar cómo se puede tolerar el cambio climático, plantear la adecuación de los límites del sitio y valorar las posibles amenazas. Muchos sitios se enfrentan a presiones que hacen peligrar sus valores. En algunos casos, el impacto puede ser gradual, en otros casos, el daño puede ser repentino e inesperado. Más allá de los efectos físicos, el cambio climático afectará a las sociedades, su actividad económica y sus relaciones.

Unos manifiestan que la solución es desarrollar el índice de vulnerabilidad climática (Day et al., 2020; Mínguez, 2020), lo que permitiría a los países que administran los sitios patrimoniales comprender, controlar y abordar mejor el riesgo del cambio climático y la necesidad de desarrollar estrategias para adaptarse a los fenómenos meteorológicos extremos y en entornos extremos, que a menudo aprovechan los conocimientos y las tradiciones de las comunidades locales (Dastgerdi y Sargolini, 2019). Sin embargo, otros manifiestan que no se pueden proteger todos los sitios, especialmente en las zonas menos desarrolladas del mundo (Markham et al., 2016). Así IUCN (2017) evalúa los cambios ocurridos durante los últimos años y advierte de la probabilidad de que el número de sitios dañados por el cambio climático siga creciendo.

En las últimas décadas, Naciones Unidas ha generado herramienta de defensa de las personas y del planeta, primero con los 8 Objetivos de Desarrollo del Milenio de la Agenda 2015 (Collier y Dollar, 2002), y posteriormente la Agenda 2030, fijando los 17 Objetivos de Desarrollo Sostenible (ODS) (Naciones Unidas, 2015), donde la cultura y el patrimonio juegan un papel crítico en este logro (Hosagrahar et al., 2016). En ese contexto, el ODS 4 (educación de calidad) y ODS 11 (ciudades y comunidades sostenibles) se refieren en esencia a la salvaguardia y la protección de la cultura "garantizando una educación de calidad inclusiva y equitativa" y "defendiendo y gestionando el patrimonio cultural y natural del mundo" respectivamente (ICOMOS, 2017) y cómo a través del ODS 13 (acción por el clima) "hay que tomar medidas urgentes para combatir el cambio climático y sus efectos".

En conciencia con los cambios que acaecen en el planeta y las nuevas necesidades de la sociedad, la UNESCO creó la Lista del Patrimonio Mundial y una segunda Lista en Peligro para alertar de los que son receptores de algún tipo de amenaza contra su integridad, continuidad y conservación. La mayoría de los sitios tienen problemas relacionados con las actividades de uso del suelo (agricultura, minería o construcción), el desarrollo turístico, los conflictos armados, la gestión ineficiente de la administración o la corrupción, que son otros factores que causan el deterioro de los sitios (Caust y Vecco, 2017; Hølleland et al., 2019; Labadi, 2019).

El objetivo del trabajo es identificar los cambios espacio-temporales que la Lista en Peligro del Patrimonio Mundial ha experimentado (1979-2018) en las distintas regiones del mundo.

\section{Metodología}

Para la recopilación de información se han empleado State of Conservation Reports 2018 (UNESCO), documentos de inscripción de los lugares y diferentes informes generados de convenciones para la protección del patrimonio, con el fin de analizar las fechas más relevantes en su historia como parte de las listas de la UNESCO (fecha de declaración del lugar en la Lista del Patrimonio Mundial y fecha de entrada en la Lista en Peligro), los tipos de bienes de los que se trata, la localización de los mismos, inclusiones por razones de emergencia, retiradas del título, el desarrollo económico 
del país al que pertenecen y las asistencias de la UNESCO en forma de ayudas y subvenciones que se han otorgado a estos lugares.

\section{Resultados}

\subsection{Posicionamiento espacial}

En la actualidad casi el 5\% de los más de mil lugares que conforman la lista del Patrimonio Mundial de la UNESCO se encuentran en riesgo. El Patrimonio Mundial en Peligro ha experimentado un crecimiento significativo en las últimas décadas. Tan sólo desde el año 2010, la Lista ha visto como se han añadido hasta 30 nuevos lugares en riesgo conformando los 54 Bienes en peligro actuales.

La distribución de estos lugares es diseminada, ubicándose en 32 países diferentes. Sin embargo, es necesario matizar este dato pues se encuentra una alta concentración de los mismos en países africanos y asiáticos de Oriente Medio a causa de los conflictos armados. A pesar de esta alta dispersión, existen países que son grandes contenedores de estos lugares amenazados entre los que merecen especial mención Siria, la República Democrática del Congo y Libia con al menos 5 sitios que se sitúan precisamente en estas regiones que se señalan con alta concentración.

A lo largo de la historia del Patrimonio en Peligro, 92 bienes se han registrado en esta Lista. La figura 1 recoge su evolución y distribución espacial (Fig. 1). Con una etapa inicial de pocos espacios en peligro, y donde se empieza a vislumbrar los riesgos del patrimonio de África. Una segunda etapa muy pareja, para llegar a finales del siglo XX, donde se manifiestan el aumento exponencial de los continentes más vulnerables, es decir, África y Asia.

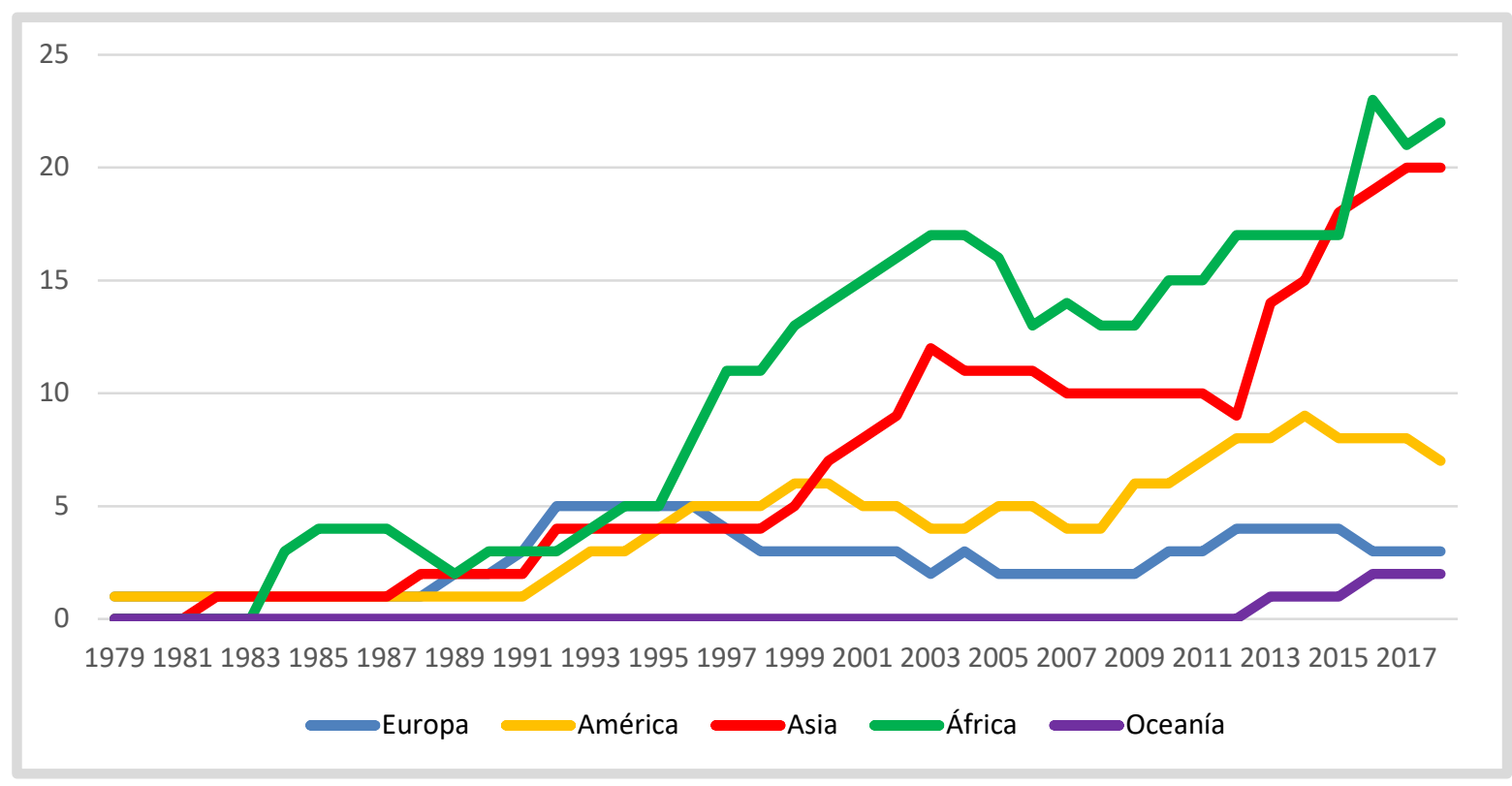

Fuente: Elaboración propia basada en Naciones Unidas (2018)

Fig. 1 Entrada de los Patrimonios en la Lista en Peligro por continentes (1979-2018)

\subsection{Posicionamiento económico}

A pesar de que se pueda pensar que los Patrimonio en Peligro están situados en los países más pobres, diversas fuentes como las Naciones Unidas (2018) o el Banco Mundial (2018) muestran que los sitios en peligro están en cualquier continente y país, aunque es cierto que las amenazas que se ciernen sobre ellos son bien distintas. Se han seleccionado tres fuentes (Naciones Unidas, Fondo Monetario Internacional y Banco Mundial). Cada uno de ellos, diferencia entre países desarrollados y en vías de desarrollo según criterios diferentes (análisis generales, criterios económicos y necesidades sociales) lo que proporciona una perspectiva más imparcial. 
Las Naciones Unidas elaboran su propia clasificación influyendo múltiples factores como son el PIB, la población, la escolaridad, las emisiones de CO2, la esperanza de vida o el INB per cápita. También, presentan el Índice de Desarrollo Humano (IDH) con una visión más social y no tan influida por la economía (salud de la población a través de su esperanza de vida al nacer, la educación mediante los años de escolarización e ingresos para evaluar los estándares de vida digna). El valor medio que separa a los países desarrollados de los que se encuentran en vías de desarrollo está en 0.550 (2018).

El Banco Mundial o el Fondo Monetario Internacional clasifican a los países con carácter económico en función de sus ingresos económicos en 4 categorías: economías de ingresos bajos (1,025 \$ o menos), medio-bajas (de 1,026 \$ a 3,995 \$), medio-altas $(1,026$ a 3,995 \$) y altos (3,996 \$ a 12,375 \$). En el presente estudio, se considerarán las dos primeras como países en vías de desarrollo y las dos últimas como países desarrollados.

En la figura 2, se pueden observar los resultados obtenidos de la puesta en común de estas diversas clasificaciones (Fig. 2).

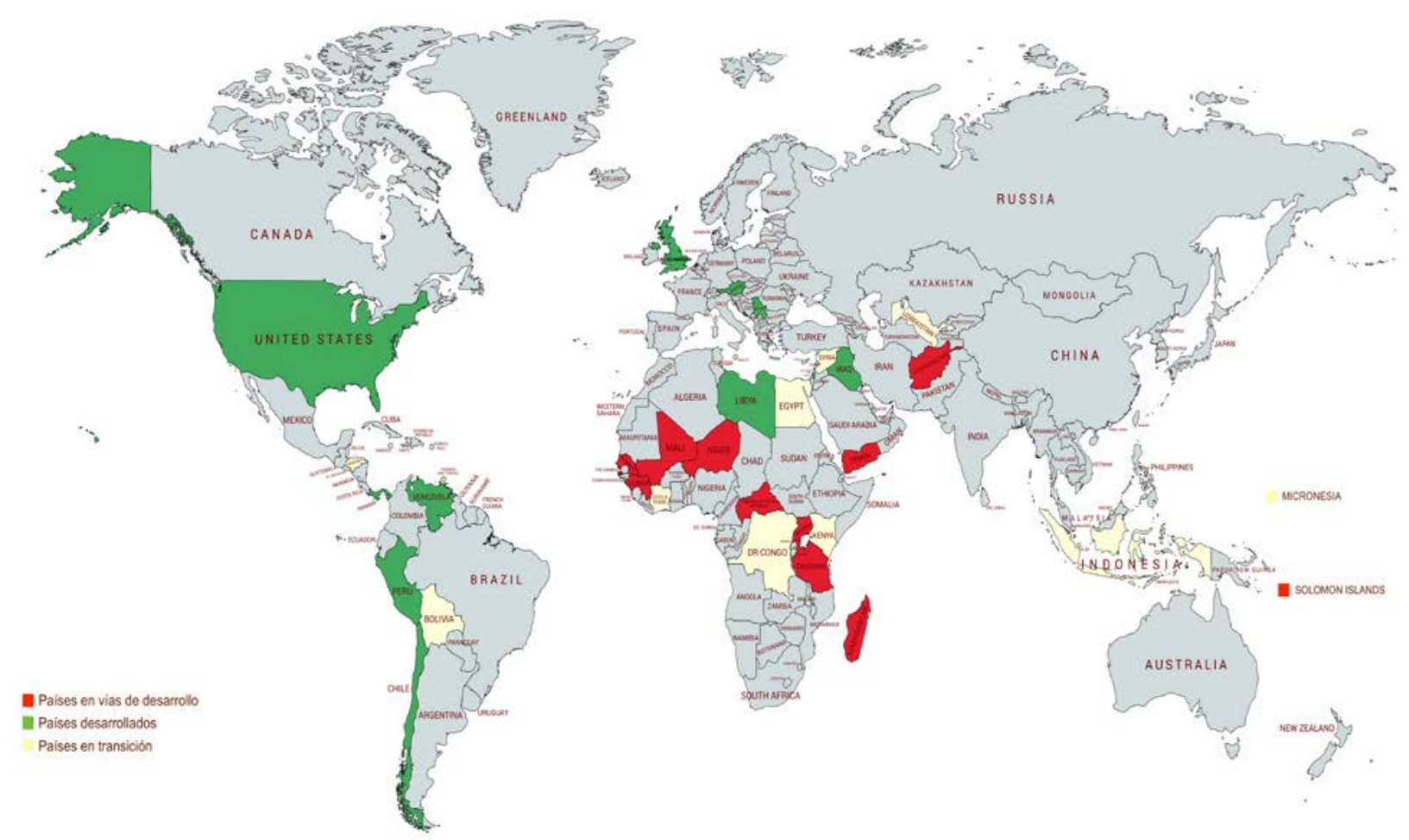

Fuente: Elaboración propia basada en Naciones Unidas (2018), Banco Mundial (2018) y Fondo Monetario Internacional (2018)

Fig. 2 Nivel de desarrollo de los países con Patrimonio Mundial en Peligro (2018)

Los países desarrollados que tienen Patrimonio Mundial en Peligro son Austria, Chile, EE.UU, Irak, Libia, Palestina, Panamá, Perú, Reino Unido, Serbia y Venezuela (un 34,5\% del total). Los países en transición son aquellos que presentan necesidades sociales por su IDH. Y finalmente, los países en vías de desarrollo son principalmente los que tienen un insuficiente desarrollo económico, ya que tampoco pueden hacerse cargo de su patrimonio.

\subsection{Duración en esta situación}

El periodo de permanencia de los lugares que están en la "Lista Negra" es relativamente alto. Si bien se han establecido tramos de 5 años para el análisis, el último tramo es considerablemente alarmante, ya que algunos sitios llevan más de 15 años en esta prolongada situación. Estos periodos se justifican con otro dato que aporta la UNESCO: el estudio del nivel de riesgo de los lugares a través del TIC (Threat Intensity Coefficient) (Turner et al., 2011). Estas medidas se dirigen más a actividades de inspección y monitoreo que al espectro completo del proceso de evaluación. La presencia 
de una amenaza grave sobre un lugar se mide año por año cuando sobrepasa el conocido umbral mínimo estándar de preocupación. Si este ratio en la línea de los últimos 15 años ha registrado al menos una amenaza, se considera peligro potencial ya que en la actualidad aún es representativo por la cercanía en el tiempo.

En este caso, según el estudio realizado, un 63\% llevan menos de 10 años y un 9,25\% entre 11-15 años. Lo realmente alarmante son el $27,75 \%$ restante sobrepasan los 15 años en la lista, tiempo suficiente como para que un peligro no sea potencial y se encuentran en un estado de "acomodamiento" (Fig. 3).

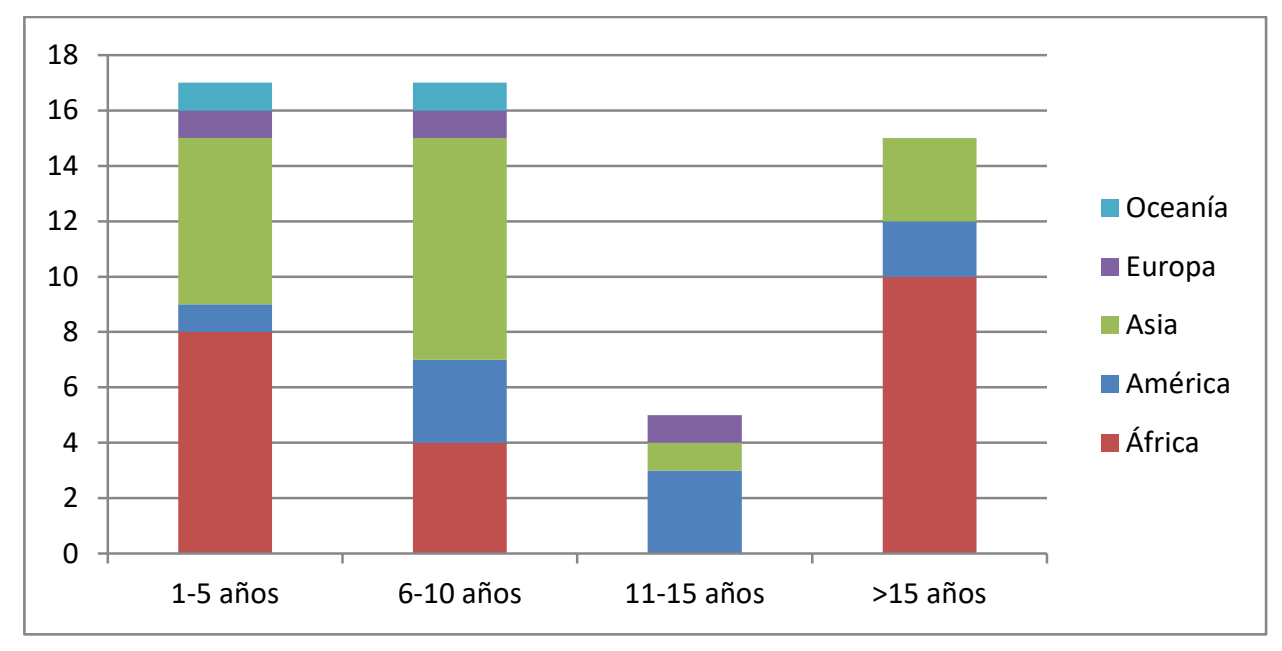

Fuente: Elaboración propia basada en UNESCO (2019)

Fig. 3 Permanencia de los BPM en la Lista en Peligro (2018)

En casi todos los tramos predominan los sitios africanos, así como la inscripción de lugares culturales sobre los naturales, salvo en este último en el que se traspasa el umbral de los 15 años. Dentro de éstos se incluyen las Reservas Naturales del Monte Nimba y de Air y Teneré, la Reserva de fauna Okapi y los Parques Nacionales de ManovoGounda, Virunga, Garamba, Kahuzi-Biega y Salonga.

\subsection{Ayudas para salir de esta situación}

Aunque la "Lista Negra", según Bertacchini et al. (2011) y Caust \& Vecco (2017), sirve como elemento disciplinario señalando y avergonzando a los estados que no cumplen las reglas, otro de los fines principales de la UNESCO es ayudar a estos lugares que, por diferentes motivos, se encuentran en situaciones amenazantes. A través de la alerta a la comunidad internacional, con la inclusión en la lista se centra el foco en ellos y, por otro lado, el aporte de ayuda financiera a los mismos colabora en el proceso de devolver a estos lugares excepcionales una situación estable.

En los últimos años se han otorgado un total de 200 ayudas a los 54 bienes en peligro actuales. Tan sólo un 18.5\% no ha recibido ninguna ayuda, y los propios países han tenido que hacerse cargo de los gastos. Sin embargo, el 55.5\% ha acogido entre 1-5 ayudas, principalmente en Asia (Oriente Medio) y África, el 22\% entre 6-10 ayudas concentradas en el continente africano, y un $4 \%$ ha recibido más de 10 ayudas. En la mayoría de las ocasiones han tenido una cuantía media de 24.593 USD moviéndose en una franja de entre 167 USD y 150.000 USD.

En este último caso, los más necesitados de ayuda han sido el Parque Nacional de Garamba y la Reserva Natural del Monte Nimba. El Parque Nacional de Garamba ha sido receptora de 14 de las cuales 7 fueron en ocasiones de emergencia. El estado del Parque ha sido foco de preocupación debido a que posee gran variedad fauna en peligro de extinción como es el caso del rinoceronte blanco y la poco común jirafa del Congo. Amenazados por guerrillas y por la caza furtiva, ha sido difícil conocer con exactitud el estado tanto de los animales como del Parque. La Reserva Natural del Monte Nimba ha contabilizado hasta 20 en su gran mayoría de conservación (16) siendo receptora de casi medio millón de USD en total. Es el único lugar en peligro transfronterizo encontrándose entre Guinea y Costa de Marfil, y se encuentra amenazado por el proyecto de concesión de una mina de hierro a un consorcio internacional y por la instalación de gran número de refugiados en el Parque. 
La UNESCO ha aportado desde 1980, 4.918.658 USD destinados a asistencias al patrimonio en peligro que en 2019 conforma la Lista en Riesgo. Pueden distinguirse tres tipos de asistencias: conservación, preparación y emergencia. El $64,50 \%$ de las asistencias han sido para fines de conservación (aprovisionamiento de equipamiento, exposiciones fotográficas, etc.), el 22\% para situaciones de emergencia (rescate de especies en extinción) y sólo el 13,50\% se adjudicaron a la preparación del lugar (reuniones, material de trabajo, desarrollo de cooperación, etc.).

Como se puede observar en la figura 4, la evolución de las asistencias ha seguido un desarrollo irregular en el que identifican grandes tramos de tiempo en los que no se han adjudicado subvenciones de preparación entre los que destacan los últimos 11 años (Fig. 4). Por otro lado, ha habido años en los que las emergencias han sido punteras como desde 1997 al 2001 y del 2010 al 2016 en el que se dieron casi medio millón de USD destinadas a esta causa. En cuanto a la conservación se observan grandes altibajos a lo largo de los años y una carencia especial durante los años de la crisis.

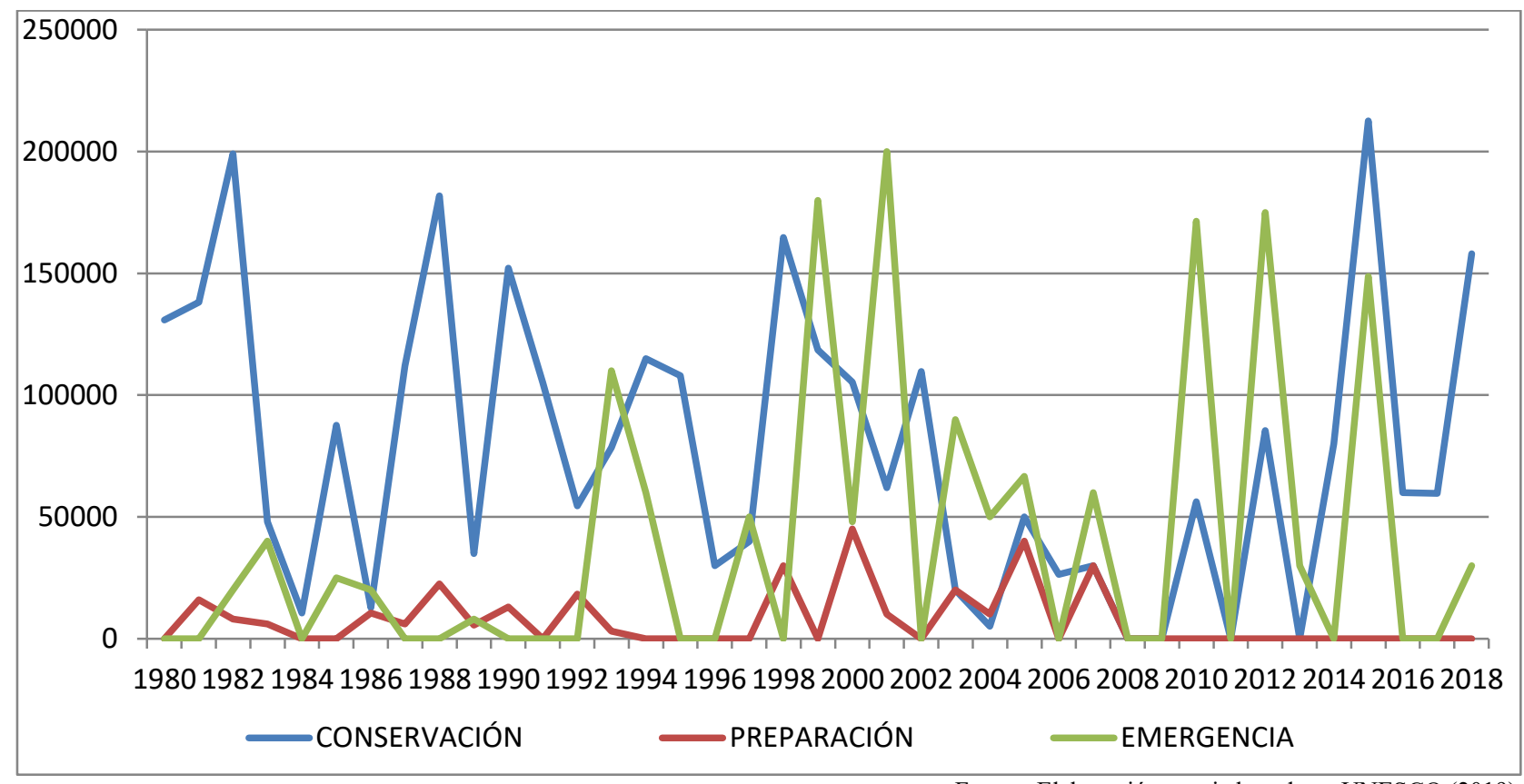

Fuente: Elaboración propia basada en UNESCO (2019)

Fig. 4 Evolución de las asistencias a los BPM en Peligro (2018)

En la figura 5 se muestran las ayudas otorgadas por la UNESCO en función de los continentes receptores de las mismas (Fig. 5). Es importante peso de África como beneficiario, principalmente en la asistencia por conservación y por emergencia. Así como la ausencia de las ayudas a Europa.

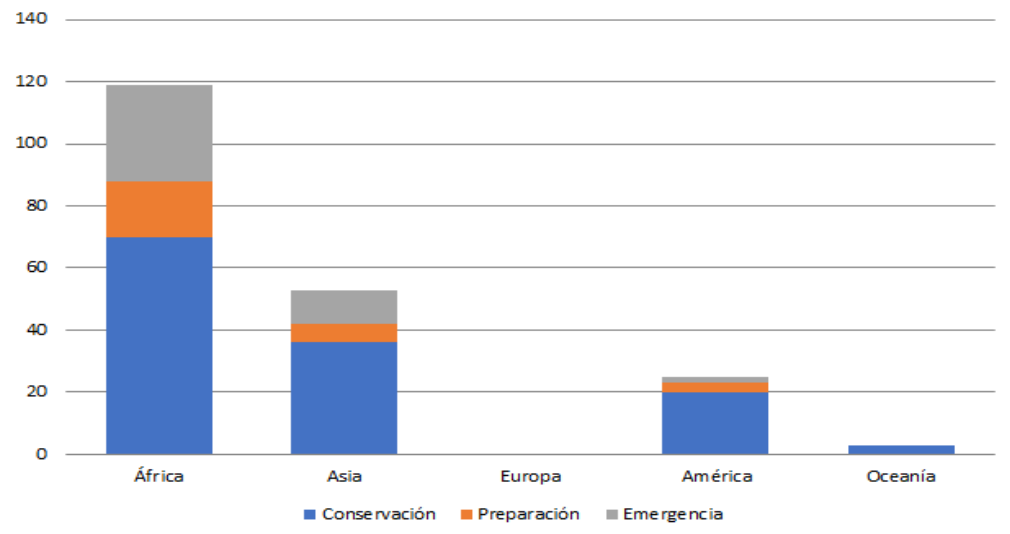

Fuente: Elaboración propia basada en UNESCO (2019)

Fig. 5 Distribución de las asistencias a los BPM en Peligro por continentes (2018) 


\section{Conclusiones}

El Patrimonio Mundial en Peligro ha experimentado un crecimiento significativo, principalmente en la última década. Los sitios en peligro están en cualquier continente. Sin embargo, la gestión de los mismos sí parece seguir un patrón y es que mientras que los países consolidados aprovechan la protección como recurso para atraer el turismo, los emergentes, al menos conservan estos lugares e intentan evitar el apoderamiento empresarial.

Se observa una dispersión de los Patrimonios Mundiales aunque se ha identificado una mayor concentración en África y Oriente Medio. Aunque la lista se actualiza anualmente, existe una gran proporción de lugares que se "acomodan" en ella. La UNESCO es proactiva y se adapta a las singularidades de cada caso.

El periodo de permanencia de los lugares que están en la "Lista Negra" es relativamente alto. El 27,75\% de los bienes sobrepasan los 15 años en la lista.

\section{Referencias}

Allan, J. R., Venter, O., Maxwell, S., Bertzky, B., Jones, K., Shi, Y., y Watson, J. E. M. (2017). Recent Increases in Human Pressure and Forest Loss Threaten Many Natural World Heritage Sites. Biological Conservation, 206, 47-55. https://doi.org/10.1016/j.biocon.2016.12.011

Bertacchini, E., Saccone, D., y Santagata, W. (2011). Embracing diversity, correcting inequalities: towards a new global governance for the UNESCO World Heritage. International journal of cultural policy, 17(3), $278-288$. https://doi.org/10.1080/10286632.2010.528833

Caust, J., y Vecco, M. (2017). Is UNESCO World Heritage recognition a blessing or burden? Evidence from developing Asian countries. Journal of Cultural Heritage, 27, 1-9. https://doi.org/10.1016/j.culher.2017.02.004

Collier, P., y Dollar, D. (2002). Aid allocation and poverty reduction. European economic review, 46(8), 1475-1500.

Dastgerdi, A. S., y Sargolini, M. (2019). Vulnerability Assessment and Conservation of Heritage Sites in A Changing Climate. International Journal of Landscape Architecture Research 3(2), 121-129.

Day, J. C., Heron, S. F., y Markham, A. (2020). Assessing the climate vulnerability of the world's natural and cultural heritage. Parks Stewardship Forum 36(1), 144-153.

Hall, C. M., Baird, T., James, M., y Ram, Y. (2016). Climate change and cultural heritage: conservation and heritage tourism in the Anthropocene. Journal of Heritage Tourism, 11(1), 10-24. http://doi.org/10.1080/1743873X.2015.1082573

Harvey, D. y Perry, J. (2015). The future of Heritage as Climates Change: Loss, Adaptation and Creativity. Nueva York: Routledge.

Hølleland, H., Hamman, E., y Phelps, J. (2019). Naming, shaming and fire alarms: the compilation, development and use of the list of World Heritage in danger. Transnational Environmental Law, 8(1), 35-57. https://doi.org/10.1017/S2047102518000225

Hosagrahar, J., Soule, J., Girard, L. F., y Potts, A. (2016). Cultural heritage, the UN sustainable development goals, and the new urban agenda. BDC. Bollettino Del Centro Calza Bini, 16(1), 37-54. https://doi.org/10.6092/2284-4732/4113

ICOMOS. (2017). ICOMOS Action Plan: Cultural Heritage and Localizing the UN Sustainable Development Goals (SDGs). Recuperado el 8 de enero de 2020 de https://www.icomos.org/images/DOCUMENTS/Secretariat/2017/ICOMOS_Action_Plan_ Cult_Heritage_and_Localizing_SDGs_20170721.pdf

IUCN. (2017). Perspectiva del Patrimonio Mundial de la UICN 2: Evaluación sobre la conservación de todos los sitios naturales del Patrimonio Mundial. https://doi.org/10.2305/IUCN.CH.2017.17.es

Labadi, S. (2019). Re-examining World Heritage and sustainable development. Routledge Companion of Global Heritage Conservation. Londres: Routledge.

Markham, A., Osipova, E., Lafrenz Samuels, K., y Caldas, A. (2016). World Heritage and tourism in a changing climate. París: UNESCO Publishing.

Maus, S. (2014). Hand in hand against climate change: Cultural human rights and the protection of cultural heritage. Cambridge Review of International Affairs, 27, 699-716. https://doi.org/10.1080/09557571.2014.960811

Mínguez, B. (2020). Resilient cultural heritage for a future of climate change. Journal of International Affairs, 73(1), 101-120. https://doi.org/10.2307/26872781 
Naciones Unidas. (2015). Transforming our world. The 2030 agenda for sustainable development. Recuperado el 19 de diciembre de 2019 de http://sustainabledevelopment.un.org/

Turner, M., Pereira, A., y Patry, M. (2011). Revealing the level of tension between cultural heritage and development in World Heritage cities-part 2. En Proceedings of the 6th International Seminar on Urban Conservation: Measuring Heritage Conservation Performance, Recife (pp. 124-133).

UNESCO. (2017). Climate Change and World Heritage. Recuperado el 21 de diciembre de 2019 de http://whc.unesco.org/en/activities/883/. 